\title{
The average tree solution for cooperative games with communication structure
}

Citation for published version (APA):

Herings, P. J. J., van der Laan, G., Talman, A. J. J., \& Yang, Z. (2008). The average tree solution for cooperative games with communication structure. METEOR, Maastricht University School of Business and Economics. METEOR Research Memorandum No. 026 https://doi.org/10.26481/umamet.2008026

Document status and date:

Published: 01/01/2008

DOI:

10.26481/umamet.2008026

Document Version:

Publisher's PDF, also known as Version of record

\section{Please check the document version of this publication:}

- A submitted manuscript is the version of the article upon submission and before peer-review. There can be important differences between the submitted version and the official published version of record.

People interested in the research are advised to contact the author for the final version of the publication, or visit the DOI to the publisher's website.

- The final author version and the galley proof are versions of the publication after peer review.

- The final published version features the final layout of the paper including the volume, issue and page numbers.

Link to publication

\footnotetext{
General rights Owners
rights.

- You may freely distribute the URL identifying the publication in the public portal. please follow below link for the End User Agreement:

www.umlib.nl/taverne-license

Take down policy

If you believe that this document breaches copyright please contact us at:

repository@maastrichtuniversity.nl

providing details and we will investigate your claim.
}

Copyright and moral rights for the publications made accessible in the public portal are retained by the authors and/or other copyright owners and it is a condition of accessing publications that users recognise and abide by the legal requirements associated with these

- Users may download and print one copy of any publication from the public portal for the purpose of private study or research.

- You may not further distribute the material or use it for any profit-making activity or commercial gain

If the publication is distributed under the terms of Article $25 \mathrm{fa}$ of the Dutch Copyright Act, indicated by the "Taverne" license above, 
P. Jean-Jacques Herings, Gerard van der Laan, Dolf Talman, Zaifu Yang

The Average Tree Solution for Cooperative Games with Communication Structure

$\mathrm{RM} / 08 / 026$

JEL code: C71

\section{METE@R}

Maastricht research school of Economics of TEchnology and ORganizations

Universiteit Maastricht

Faculty of Economics and Business Administration P.O. Box 616

NL - 6200 MD Maastricht

phone : ++31433883830

fax : ++31433884873 


\title{
The Average Tree Solution for Cooperative Games with Communication Structure*
}

\author{
P. Jean-Jacques Herings,${ }^{\dagger}$ Gerard van der Laan, ${ }^{\ddagger}$ Dolf Talman,,${ }^{\S}$ Zaifu Yang $₫$
}

September 3, 2008

*An earlier version of this paper was presented as "The average tree solution for cooperative games with limited communication structure" at the World Congress of the Game Theory Society, July 2008, Evanston. The first and the forth authors would like to thank the Netherlands Organisation for Scientific Research (NWO) and CentER of Tilburg University for financial support.

${ }^{\dagger}$ P.J.J. Herings, Department of Economics, Maastricht University, P.O. Box 616, 6200 MD Maastricht, The Netherlands, e-mail: P.Herings@algec.unimaas.nl.

${ }^{\ddagger}$ G. van der Laan, Department of Econometrics and Tinbergen Institute, Vrije Universiteit, De Boelelaan 1105, 1081 HV Amsterdam, The Netherlands, e-mail: glaan@feweb.vu.nl.

$\S$ A.J.J. Talman, CentER, Department of Econometrics \& Operations Research, Tilburg University, P.O. Box 90153, 5000 LE Tilburg, The Netherlands, e-mail: talman@uvt.nl.

IZ. Yang, Faculty of Business Administration, Yokohama National University, Yokohama 2408501, Japan. e-mail: yang@ynu.ac.jp. 


\begin{abstract}
We study cooperative games with communication structure, represented by an undirected graph. Players in the game are able to cooperate only if they can form a network in the graph. A single-valued solution, the average tree solution, is proposed for this class of games. Given the graph structure we define a collection of spanning trees, where each spanning tree specifies a particular way by which players communicate and determines a payoff vector of marginal contributions of all the players. The average tree solution is defined to be the average of all these payoff vectors. It is shown that if a game has a complete communication structure, then the proposed solution coincides with the Shapley value, and that if the game has a cycle-free communication structure, it is the solution proposed by Herings, van der Laan and Talman (2008). We introduce the notion of linkconvexity, under which the game is shown to have a non-empty core and the average tree solution lies in the core. In general, link-convexity is weaker than convexity. For games with a cycle-free communication structure, link-convexity is even weaker than super-additivity.
\end{abstract}

Keywords: Cooperative game, graph structure, single-valued solution, core, convexity, spanning tree.

AMS subject classification: 90B18, 91A12, 91A43.

JEL code: C71. 


\section{Introduction}

A situation in which sets of players can realize joint payoffs by cooperating can be formulated as a cooperative game $(N, v)$, where $N=\{1, \ldots, n\}$ is a finite set of players and $v: 2^{N} \rightarrow \mathbb{R}$ a characteristic function with $v(S)$ the joint payoff that the players in $S \subset N$ can obtain by cooperation. In the standard approach it is assumed that any coalition $S$ can form and achieve worth $v(S)$. However, there are many situations of interest where cooperation among people depends on how they can communicate and coordinate.

In a seminal paper, Myerson [13] formulates such games with communication structure by a triple $(N, v, L)$, where $N$ is a set of players, $v: 2^{N} \rightarrow \mathbb{R}$ a characteristic function, and $L \subset\{\{i, j\} \mid i, j \in N, i \neq j\}$ a set of edges on $N$ representing communication links between players. A coalition $S$ can only cooperate if the set of nodes $S$ is connected in the graph $(N, L)$ and thus any two players in $S$ can communicate with each other, either directly or indirectly through other players in $S$. Myerson also proposes a single-valued solution for games with communication structure. Nowadays this solution is known as the Myerson value. The Myerson value of a game with communication structure equals the Shapley value of the so-called Myerson restricted game, induced by the communication structure, and is characterized by component efficiency and fairness.

Alternative characterizations of the Myerson value are given in Myerson [14] and Borm, Owen, and Tijs [2]. In the latter paper also another solution for games with communication structure has been proposed, the so-called positional value, see also Meessen [12]. This value is characterized by component efficiency and balanced total threats, see Slikker [17]. Recently, Herings, van der Laan, and Talman [9] introduced a new solution for the class of games with cycle-free communication structure, the so-called average tree solution. This solution is characterized by component efficiency and component fairness. The average tree solution lies in the core if the characteristic function $v$ is superadditive. This property does not hold for the Myerson value and the positional value.

In this paper we aim to generalize the average tree solution to the class of all games with communication structure. A tree $(N, T)$ is a cycle-free directed graph, with $T$ a collection of $n-1$ directed edges, such that for exactly one node, the root, there exists a unique directed path in $(N, T)$ to every other node. To generalize the average tree solution to the class of all games with communication structure, we define for every graph $(N, L)$ a collection of admissible spanning trees on the graph. A spanning tree is admissible if each player has exactly one successor in each component of his subordinates. Such a spanning tree describes how in the graph a player can communicate with other players in such a way that two players cannot communicate with each other if one is not a subordinate of the other. The payoff of a player in a given admissible spanning tree is then the marginal contribution of that player when he joins his subordinates, and the proposed average tree 
solution is the average of the payoff vectors for all admissible spanning trees.

We show that for games with cycle-free communication structure the average tree solution coincides with the average tree solution in Herings et al. [9] and that for games with complete communication structure the average tree solution coincides with the Shapley value. In this way the average tree solution is a proper generalization of both the Shapley value for games with full communication and the average tree solution for games with cycle-free communication structure. The number of admissible spanning trees depends on the structure of the graph. Typically, when there are more cycles, there are more ways for players to communicate and the number of admissible spanning trees becomes larger. In a cycle-free graph $(N, L)$ with $n$ players there are exactly $n$ admissible spanning trees, while if the graph is complete there are $n$ ! admissible spanning trees. So, the average tree solution is the average of a certain number of marginal vectors of the induced restricted game, where the number depends on the communication structure. It therefore differs from the Myerson value, which for every game with communication structure is given by the average of all $n$ ! marginal vectors of the restricted game.

In this paper we also introduce the notion of link-convexity for games with communication structure. For games with complete communication structure, the notion of link-convexity coincides with convexity, but in general the notion of link-convexity is weaker than convexity. For games with cycle-free communication structure, link-convexity is even weaker than superadditivity. It is well known that for convex games the Shapley value lies in the core and so the Myerson value lies in the core of the game when the (restricted) game is convex. We show that for arbitrary games with communication structure, the average tree solution is in the core if the game is link-convex. This confirms the result of Herings et al. [9] for a game with cycle-free communication structure that the average tree solution is in the core if the game is superadditive. Talman and Yamamoto [18] give for games with cycle-free communication structure a condition that is even weaker than link-convexity to guarantee that the average tree solution is in the core. We also illustrate that the Myerson value may not be in the core if the game is link-convex but not convex.

This paper is organized as follows. Section 2 is a preliminary section on games with communication structure. In Section 3 the average tree solution for all games with communication structure is introduced. In Section 4 the classes of cycle-free and complete communication structures are discussed. In Section 5 the notion of link-convexity is introduced and it is shown that the average tree solution lies in the core if the game is link-convex. Section 6 concludes. 


\section{TU-games with communication structure}

A transferable utility cooperative game with communication structure is represented by $(N, v, L)$ with $N=\{1, \ldots, n\}$ a finite set of players, $v: 2^{N} \rightarrow \mathbb{R}$ a characteristic function, and $(N, L)$ an undirected graph with node set the set of players $N$ and a set of edges $L$, being a subset of $\{\{i, j\} \mid i \neq j, i, j \in N\}$. The set $L$ stands for the collection of communication links between players. If $\{i, j\}$ belongs to $L$, then players $i$ and $j$ are able to communicate with each other.

A sequence of different nodes $\left(i_{1}, \ldots, i_{k^{\prime}}\right)$ is called a path from $i_{1}$ to $i_{k^{\prime}}$ in the graph $(N, L)$ if $\left\{i_{k}, i_{k+1}\right\} \in L$ for $k=1, \ldots, k^{\prime}-1$. A coalition of players $S \in 2^{N}$ forms a network in the graph $(N, L)$ if $S$ is connected in the graph, i.e., for any $i, j \in S, i \neq j$, there is a path in $S$ from $i$ to $j$. Notice that the empty set and all singleton coalitions are networks by definition. Any two members in a network are able to communicate with each other directly or indirectly through other players in the same network. A coalition $S$ of players is called a component in the graph $(N, L)$ if $S$ forms a network and $S$ cannot form a larger network with any other player $j \in N \backslash S$. A sequence of at least three different nodes $\left(i_{1}, \ldots, i_{k^{\prime}}\right)$ is called a cycle in the graph $(N, L)$ if $(1)$ it is a path in $(N, L)$ and $(2)$ $\left\{i_{k^{\prime}}, i_{1}\right\} \in L$. A graph $(N, L)$ is cycle-free if it does not contain any cycle. For given graph $(N, L)$, each $K \in 2^{N}$ induces the subgraph $(K, L(K))$, with $L(K)=\{\{i, j\} \in L \mid i, j \in K\}$ the set of links on $K$. Notice that $L(N)=L$. The concepts defined above for the graph $(N, L)$ are defined similarly for $(K, L(K))$. For $K \in 2^{N}$, let $C^{L}(K)$ denote the collection of all networks in the graph $(K, L(K))$ and let $\widehat{C}^{L}(K)$ denote the collection of all components in $(K, L(K))$.

A directed graph on $N$ is a pair $(N, D)$ such that $D \subset\{(i, j) \in N \times N \mid i \neq j\}$ is a collection of directed edges. A player $i$ is a predecessor of $j$ and $j$ a successor of $i$ in $D$ if $(i, j) \in D$. A sequence of different nodes $\left(i_{1}, \ldots, i_{k^{\prime}}\right)$ is called a directed path from $i_{1}$ to $i_{k^{\prime}}$ in the directed graph $(N, D)$ if $\left(i_{k}, i_{k+1}\right) \in D$ for $k=1, \ldots, k^{\prime}-1$. A tree $(N, T)$ is a directed graph, with $T$ a collection of exactly $n-1$ directed edges, such that from exactly one node, called the root, there is a unique directed path to every other node. In a tree the root has no predecessor and any other player has exactly one predecessor. On the other hand, a player may have multiple successors. A player $j$ is a subordinate of $i$ in $T$ if $T$ contains a directed path from $i$ to $j$. Given an undirected graph $(N, L)$, a tree $(N, T)$ is a spanning tree of $(N, L)$ if $(i, j) \in T$ implies $\{i, j\} \in L$, i.e., any directed edge in $T$ is an undirected edge in $L$.

In the game $(N, v, L)$, a coalition $S$ of players can only cooperate and realize its worth $v(S)$ if $S$ forms a network. In the rest of the paper, we assume without loss of generality that $N$ is connected, so $N$ itself forms a network and can realize its worth $v(N)$. Otherwise, the analysis can be done analogously for each component in the graph $(N, L)$. 
When each pair of players can communicate directly, i.e., $L=\{\{i, j\} \mid i \neq j, i, j \in N\}$, $(N, v, L)$ is said to be a game with complete communication structure and often shortly denoted by $(N, v)$.

A payoff vector $x \in \mathbb{R}^{n}$ of $(N, v, L)$ is an $n$-dimensional vector giving a payoff $x_{i} \in \mathbb{R}$ to every player $i \in N$. We write $x(S)=\sum_{i \in S} x_{i}$ for $S \in C^{L}(N)$. A payoff vector $x$ is efficient if $x(N)=v(N)$, i.e., it fully distributes the worth $v(N)$ of the grand coalition $N$ to all its members. A solution for games with communication structure is a mapping $F$ that assigns to every game with communication structure $(N, v, L)$ a set of payoff vectors $F(N, v, L) \subset \mathbb{R}^{n}$. A solution $F$ is efficient if for any $(N, v, L)$ every element of $F(N, v, L)$ is efficient.

The best-known set-valued solution for games $(N, v)$ is the core, see Gillies [6], which assigns to every game $(N, v)$ the set $C(N, v)=\left\{x \in \mathbb{R}^{n} \mid x(N)=v(N)\right.$, and $x(S) \geq$ $v(S)$, for all $\left.S \in 2^{N}\right\}$ of undominated efficient payoff vectors. With full communication, a payoff vector $x$ is dominated if there exists a coalition $S$ such that $x(S)<v(S)$. However, for games with communication structure $(N, v, L)$, a coalition $S$ can only cooperate if it forms a network and so a payoff vector $x$ can only be dominated by networks. Consequently, for games with communication structure $(N, v, L)$ the core becomes equal to the set $C(N, v, L)$ given by

$$
C(N, v, L)=\left\{x \in \mathbb{R}^{n} \mid x(N)=v(N), \text { and } x(S) \geq v(S) \text {, for all } S \in C^{L}(N)\right\},
$$

i.e., the core is the set of efficient payoff vectors that are not dominated by any network $S$. Clearly, it holds that $C(N, v, L)$ is equal to $C\left(N, v^{L}\right)$, where $v^{L}$ is the characteristic function of the Myerson restricted game $\left(N, v^{L}\right)$ induced by $(N, v, L)$ and defined for $S \in 2^{N}$ by

$$
v^{L}(S)=\sum_{K \in \widehat{C}^{L}(S)} v(K)
$$

i.e., the value of coalition $S$ equals the sum of the values of its components in $(S, L(S))$.

The best-known single-valued solution for games $(N, v)$ is the Shapley value, see Shapley [16], which assigns to every game $(N, v)$ the average $\phi(N, v)$ of all $n$ ! marginal vectors $m^{\pi}(v) \in \mathbb{R}^{n}$ of the game $(N, v)$, where $\pi=(\pi(1), \ldots, \pi(n))$ is a permutation $\pi: N \rightarrow N$ assigning a unique number $\pi(i) \in N$ to every player $i \in N$ and $m^{\pi}(v)=$ $\left(m_{1}^{\pi}(v), \ldots, m_{n}^{\pi}(v)\right)$ with, for every $j \in N, m_{j}^{\pi}(v)=v\left(\pi^{j} \cup\{j\}\right)-v\left(\pi^{j}\right)$ and $\pi^{j}=\{i \in N \mid$ $\pi(i)<\pi(j)\}$. The Myerson value, see Myerson [13], is a single-valued solution assigning to every $(N, v, L)$ the Shapley value $\phi\left(N, v^{L}\right)$ of the Myerson restricted game. 


\section{The average tree solution}

In this section we present a new single-valued solution for games with communication structure. The new solution generalizes the average tree solution for games with cycle-free communication structure as introduced in Herings et al. [9]. For a game with cycle-free communication structure $(N, v, L)$ the average tree solution is the average of $n$ specific payoff vectors. More precisely, each payoff vector corresponds to one player and this vector is determined by the unique spanning tree $(N, T)$ for the cycle-free graph $(N, L)$ in which that player is the root of the tree. On the class of games with cycle-free communication structure the average tree solution has been axiomatized by component efficiency and component fairness. In [9] it is also shown that if the characteristic function of the game satisfies superadditivity, the average tree solution lies in the core $C(N, v, L)$.

To extend and generalize the average tree solution to the class of all games with communication structure, first notice that when a graph $(N, L)$ is not cycle-free, not all links are needed to communicate. For a particular player $i$, every spanning tree on $(N, L)$ having player $i$ as root describes a possibility in which player $i$ is able to communicate with the other players. We only consider spanning trees in which any player is linked to just one successor in every component of the set of his subordinates. To describe this class of spanning trees, we first give the definition of an admissible $n$-tuple of coalitions.

Definition 3.1 For given graph $(N, L)$, an $n$-tuple $B=\left(B_{1}, \ldots, B_{n}\right)$ of $n$ subsets of $N$ is admissible if it satisfies the following conditions:

(1) For all $i \in N, i \in B_{i}$, and for some $j \in N, B_{j}=N$;

(2) For all $i \in N$ and $K \in \widehat{C}^{L}\left(B_{i} \backslash\{i\}\right)$, we have $K=B_{j}$ and $\{i, j\} \in L$ for some $j \in N$.

Condition (2) of Definition 3.1 states that for every $i \in N$ each component in the subgraph $\left(B_{i} \backslash\{i\}, L\left(B_{i} \backslash\{i\}\right)\right)$ is equal to $B_{h}$ for some player $h$ being linked to player $i$. The same condition also implies that every set $B_{i}$ is a network.

Given an admissible $n$-tuple of coalitions $B$, we interpret $B_{i}$ as the set of subordinates of player $i$ together with player $i$ himself. We define the directed graph $\left(N, T^{B}\right)$ as

$$
T^{B}=\left\{(i, j) \mid B_{j} \in \widehat{C}^{L}\left(B_{i} \backslash\{i\}\right), i \in N\right\} .
$$

The notion of admissible $n$-tuples has the following properties.

Lemma 3.2 For a graph $(N, L)$, let $B$ be an admissible $n$-tuple of coalitions. Then the following properties hold. 
(1) There exists a unique player $i \in N$ such that $B_{i}=N$.

(2) For all $i, j \in N, i \neq j$, either $B_{i} \subset B_{j} \backslash\{j\}$, or $B_{j} \subset B_{i} \backslash\{i\}$, or both $B_{i} \cap B_{j}=\emptyset$ and $B_{i} \cup B_{j} \notin C^{L}(N)$;

(3) The directed graph $\left(N, T^{B}\right)$ is a spanning tree.

Proof. From Condition (1) of Definition 3.1 it follows that $B_{i}=N$ for some $i \in N$. By Condition (2) of Definition 3.1, for every $K \in \widehat{C}^{L}\left(B_{i} \backslash\{i\}\right)$ there exists $j \in N$ such that $K=B_{j}$ and $\{i, j\} \in L$, which leads to edges $(i, j)$ of $T^{B}$. Next we continue this procedure with every $j$ chosen in the previous step for which the network $B_{j}$ is not a singleton. We proceed in this way until all remaining networks are singletons. It follows immediately that $T^{B}$ is a spanning tree, which proves (3). Observe that, for all $j \in N, B_{j} \backslash\{j\}$ is the set of subordinates of player $j$ in the spanning tree $T^{B}$. Therefore, there is a unique $i \in N$ for which $B_{i}=N$, which proves (1).

To prove (2) consider two nodes $i$ and $j$. Because $T^{B}$ is a spanning tree, either $B_{i} \subset B_{j} \backslash\{j\}$ or $B_{j} \subset B_{i} \backslash\{i\}$ or $B_{i} \cap B_{j}=\emptyset$. It remains to be shown that $B_{i} \cup B_{j}$ is not a network in the last case. Since $T^{B}$ is a spanning tree, there is $j^{\prime} \neq i, j$ such that $B_{i} \subset B_{j^{\prime}}$ and $B_{j} \subset B_{j^{\prime}}$. Let $B_{j^{\prime}}$ be the minimal set with these properties. Moreover, there is no $\left(j^{\prime}, j^{\prime \prime}\right) \in T^{B}$ such that both $B_{i} \subset B_{j^{\prime \prime}}$ and $B_{j} \subset B_{j^{\prime \prime}}$, since otherwise $B_{j^{\prime \prime}}$ is a proper subset of $B_{j^{\prime}}$. It follows that $B_{i}$ and $B_{j}$ belong to different components of $\widehat{C}^{L}\left(B_{j^{\prime}} \backslash\left\{j^{\prime}\right\}\right)$, so $B_{i} \cup B_{j}$ is not connected.

Property (2) of Lemma 3.2 says that if $B_{i}$ and $B_{j}$ are two different components in $\widehat{C}^{L}\left(B_{k} \backslash\{k\}\right)$ for some $k \in N$, then there is no link in the graph $(N, L)$ between any player of $B_{i}$ and any player of $B_{j}$. This means that for any two players, if in $T^{B}$ one player is not a subordinate of the other, they cannot communicate directly with each other in the graph $(N, L)$. In the spanning tree $T^{B}$, the root player $i$ communicates with a subordinate $h$ in $T^{B}$ through his successor $j$ in the component $K$ of $\widehat{C}^{L}(N \backslash\{i\})$ that contains $h$. Then $B_{j}=K$ and on his turn, player $j$ communicates with his subordinates through his successors in the components in $\widehat{C}^{L}\left(B_{j} \backslash\{j\}\right)$, and so on.

The following example illustrates the concept of admissible $n$-tuples and their induced spanning trees.

Example 3.3 Let $(N, L)$ be given by $N=\{1,2,3,4\}$ and $L=\{\{1,2\},\{2,3\},\{3,4\},\{1,4\}\}$, i.e., $L$ is given by the cycle $(1,2,3,4)$. Consider the case where $B_{1}=N$. By Condition (2) of Definition 3.1, for the unique component $K=\{2,3,4\}$ of the subgraph on $\{2,3,4\}$, there exists a player $i$ such that $\{1, i\} \in L$ and $B_{i}=K$. Clearly, it holds that $i=2$ or 
$i=4$. First, take $i=2$, then $B_{2}=\{2,3,4\}$. For the unique component $K^{\prime}=\{3,4\}$ of the subgraph on $B_{2} \backslash\{2\}=\{3,4\}$, there is a player $j$ such that $\{2, j\} \in L$ and $B_{j}=K^{\prime}$. Clearly, $j=3$ and it follows that $B_{3}=\{3,4\}$. Finally $B_{4}=B_{3} \backslash\{3\}$, so $B_{4}=\{4\}$ is a leaf. Analogously, when $i=4$ is taken, $B_{4}=\{2,3,4\}, B_{3}=\{2,3\}$, and $B_{2}=\{2\}$.

So, there are two admissible $n$-tuple of coalitions with $B_{1}=N$, namely (i) $B_{1}=$ $N, B_{2}=\{2,3,4\}, B_{3}=\{3,4\}, B_{4}=\{4\}$, and (ii) $B_{1}=N, B_{2}=\{2\}, B_{3}=\{2,3\}$, $B_{4}=\{2,3,4\}$. By symmetry, for any $i \in N$ there are two admissible $n$-tuples of coalitions with $B_{i}=N$ and thus there are 8 admissible $n$-tuples of coalitions for the cyclic graph on $N=\{1,2,3,4\}$.

The two sets of admissible $n$-tuple of coalitions with $B_{1}=N$ induce two spanning trees with player 1 as root, the spanning tree $T_{1}=\{(1,2),(2,3),(3,4)\}$ in case $B_{2}=\{2,3,4\}$ and the spanning tree $T_{2}=\{(1,4),(4,3),(3,2)\}$ in case $B_{4}=\{2,3,4\}$. Observe that there are also two other spanning trees with player 1 as root, namely $T_{3}=$ $\{(1,2),(1,4),(2,3)\}$ and $T_{4}=\{(1,2),(1,4),(4,3)\}$, but these spanning trees do not correspond to an admissible $n$-tuple of coalitions, because player 1 has two successors in component $\{2,3,4\}$ of $\widehat{C}^{L}(N \backslash\{1\})$.

As shown in the example, the set of spanning trees induced by the collection of admissible $n$-tuples of coalitions is typically a proper subset of the collection of all spanning trees of $(N, L)$. To define the average tree solution for the class of all games with communication structure we only consider spanning trees induced by admissible $n$-tuples of coalitions. For a game with communication structure $(N, v, L)$, let $\mathcal{B}^{L}$ denote the collection of all admissible $n$-tuples of coalitions $B=\left(B_{1}, \ldots, B_{n}\right)$ for the graph $(N, L)$. Then, for every $B \in \mathcal{B}^{L}$, we define the marginal contribution vector $m^{B}(N, v, L)$, yielding a payoff $m_{i}^{B}(N, v, L)$ for every player $i \in N$.

Definition 3.4 For a game with communication structure $(N, v, L)$, the marginal contribution vector $m^{B}(N, v, L) \in \mathbb{R}^{n}$ corresponding to $B \in \mathcal{B}^{L}$ is the vector of payoffs given by

$$
m_{i}^{B}(N, v, L)=v\left(B_{i}\right)-\sum_{K \in \widehat{C}^{L}\left(B_{i} \backslash\{i\}\right)} v(K), \quad i \in N .
$$

At $m^{B}(N, v, L)$ every player $i \in N$ receives a payoff equal to the worth of network $B_{i}$ minus the total worths of the components of $\left(B_{i} \backslash\{i\}, L\left(B_{i} \backslash\{i\}\right)\right)$. With respect to the corresponding spanning tree $T^{B}$, the marginal contribution gives to every player the value of the network consisting of himself and his subordinates minus the total payoff assigned to his subordinates. Notice that a marginal contribution vector $m^{B}(N, v, L)$ is a marginal vector $m^{\pi}$ of the restricted game $\left(N, v^{L}\right)$ for any permutation $\pi$ satisfying $B_{i} \backslash\{i\} \subset \pi^{i}$ for 
all $i \in N$. We remark that spanning trees that are not induced by an admissible $n$-tuple of coalitions do not yield a payoff vector that has this property.

We now define the average tree solution as the average of all marginal contribution vectors over the collection of admissible $n$-tuples of coalitions.

\section{Definition 3.5 Average tree solution}

On the class of all games with communication structure $(N, v, L)$, the average tree $(A T)$ solution assigns the payoff vector $\operatorname{AT}(N, v, L)$ given by

$$
\operatorname{AT}(N, v, L)=\frac{1}{\left|\mathcal{B}^{L}\right|} \sum_{B \in \mathcal{B}^{L}} m^{B}(N, v, L) .
$$

The number of admissible $n$-tuples depends on the structure of the graph $(N, L)$. In the next section we discuss two special cases, cycle-free graphs and complete graphs.

\section{Special cases of games with communication struc- ture}

In this section we discuss the average tree solution for games with cycle-free communication structure and complete communication structure.

Lemma 4.1 Let $(N, L)$ be a cycle-free graph. Then for every $i \in N$ there is exactly one admissible $n$-tuple of coalitions such that $B_{i}=N$.

Proof. For some $i \in N$, take $B_{i}=N$. Since the graph is cycle-free and connected, player $i$ is linked to exactly one player in each component of $N \backslash\{i\}$. For given $K \in \widehat{C}^{L}(N \backslash\{i\})$, let $j \in K$ be the unique player such that $\{i, j\} \in L$. Then, by Condition (2) of Definition 3.1, $B_{j}=K$. Next, for each component $B_{j} \in \widehat{C}^{L}(N \backslash\{i\})$, player $j \in B_{j}$ is linked to exactly one player in each component of $\widehat{C}^{L}\left(B_{j} \backslash\{j\}\right)$. For given $K^{\prime} \in \widehat{C}^{L}\left(B_{j} \backslash\{j\}\right)$, let $j^{\prime} \in K^{\prime}$ be the unique player in $K^{\prime}$ such that $\left\{j, j^{\prime}\right\} \in K^{\prime}$. Then, again by Condition (2) of Definition 3.1, $B_{j^{\prime}}=K^{\prime}$. Continuing this procedure as long as there are components consisting of more than one player, we obtain the unique admissible $n$-tuple of coalitions with $B_{i}=N$.

Using this lemma we show that for games with cycle-free communication structure the average tree solution coincides with the solution introduced in Herings et al. [9] for this particular class of games. When $(N, L)$ is cycle-free, let $T^{i}, i \in N$, be the unique spanning 
tree with node $i$ as its root. For a game with cycle-free communication structure $(N, v, L)$, the spanning tree $T^{i}$ determines a marginal contribution vector $m^{i}(N, v, L)$ with payoff

$$
m_{j}^{i}(N, v, L)=v\left(K_{j}^{i}\right)-\sum_{\left\{j \mid\left(j, j^{\prime}\right) \in T^{i}\right\}} v\left(K_{j^{\prime}}^{i}\right), j \in N,
$$

where, for $j \in N, K_{j}^{i}$ is the set of nodes consisting of $j$ and all its subordinates in $T^{i}$. The average tree solution for games with cycle-free communication structure as introduced in Herings et al. [9] then yields the average of these $n$ marginal contribution vectors.

Theorem 4.2 For a game with cycle-free communication structure $(N, v, L)$ it holds that

$$
A T(N, v, L)=\frac{1}{n} \sum_{i \in N} m^{i}(N, v, L)
$$

Proof. By Lemma 4.1 we have that for any $i \in N$ there is a unique admissible $n$-tuple of coalitions with $B_{i}=N$. Let $B(i)$ be this $n$-tuple of coalitions. From the construction in the proof of Lemma 4.1, it follows immediately that the spanning tree $T^{B(i)}$ corresponding to $B(i)$ coincides with the unique spanning tree $T^{i}$ having $i$ as its root. Hence $m^{i}(N, v, L)=m^{B(i)}(N, v, L)$ for all $i \in N$.

Next we prove that for games with complete communication structure the average tree solution coincides with the Shapley value.

Lemma 4.3 Let $(N, L)$ be a complete graph. Then there are $n$ ! admissible $n$-tuples of coalitions.

Proof. For an arbitrarily chosen player $i_{1} \in N$, we consider the collection of all admissible $n$-tuples $B$ with $B_{i_{1}}=N$. Since the graph $(N, L)$ is complete, $N \backslash\left\{i_{1}\right\}$ is connected and thus consists of exactly one component. For any arbitrarily chosen $i_{2}$ in $N \backslash\left\{i_{1}\right\}$ we can set $B_{i_{2}}=N \backslash\left\{i_{1}\right\}$, since $i_{1}$ is connected with every other player. The network $B_{i_{2}} \backslash\left\{i_{2}\right\}$ consists of exactly one component and for every $i_{3} \in B_{i_{2}} \backslash\left\{i_{2}\right\}$ we can set $B_{i_{3}}=B_{i_{2}} \backslash\left\{i_{2}\right\}$. Continuing in this way at each step $k, k=1, \ldots, n$, we can take an arbitrarily chosen player $i_{k}$ in $B_{i_{k-1}} \backslash\left\{i_{k-1}\right\}$ and set $B_{i_{k}}=B_{i_{k-1}} \backslash\left\{i_{k-1}\right\}$, where $B_{i_{k}}=N$ when $k=1$. Since at each step, any player in the remaining set can be chosen, there are $n$ ! admissible $n$-tuples of coalitions.

Theorem 4.4 Let $(N, v, L)$ be a game with complete communication structure. Then the average tree solution is equal to the Shapley value of $(N, v)$. 
Proof. For a game $(N, v)$, the Shapley value $\phi(N, v)$ is equal to the average of all $n$ ! marginal vectors $m^{\pi}(v)$. Let $B$ be an admissible $n$-tuple of coalitions constructed in the proof of Lemma 4.3. For the player $i_{k}$ chosen at step $k$, the marginal contribution $m_{i_{k}}^{B}(N, v, L)$ is equal to $v\left(B_{i_{k}}\right)-v\left(B_{i_{k+1}}\right)$, where $B_{i_{n+1}}=\emptyset$. We define a bijection between admissible $n$-tuple of coalitions $B$ and permutations $\pi$ by assigning permutation $\pi^{B}$, given by $\pi_{i_{k}}^{B}=n+1-i_{k}, k=1, \ldots, n$, to admissible $n$-tuple of coalitions $B$. By definition of the marginal vector we have that $m^{\pi^{B}}(v)=m^{B}(N, v, L)$. Hence, the average of all marginal contribution vectors $m^{B}(N, v, L)$ is equal to the average of all marginal vectors $m^{\pi}(v)$.

Observe that for a complete graph each of the $n$ ! admissible $n$-tuples of coalitions generates a path graph, i.e., each player has exactly one successor and one predecessor, except the first chosen player who has no predecessor and the last chosen player who has no successor. On the other hand, any given path graph corresponds to the permutation in which the last chosen player enters first, the second last chosen player enters second, and so on.

\section{Core properties}

In this section we provide conditions for arbitrary games with communication structure under which the average tree solution lies in the core. For a game $(N, v)$ it is well-known that the Shapley-value $\phi(N, v)$ is in the core $C(N, v)$ if the game is convex, the requirement that $v(S)+v(T) \leq v(S \cup T)+v(S \cap T)$ for every $S, T \subset N$. A game is superadditive if these inequalities are satisfied for every $S$ and $T$ such that $S \cap T=\emptyset$. Superadditivity is insufficient to ensure that a game has a non-empty core. We say that a game with communication structure $(N, v, L)$ is superadditive if its Myerson restricted game $\left(N, v^{L}\right)$ is superadditive. It can be shown that a superadditive game with cycle-free communication structure has a non-empty core. In particular, it follows from Demange [5] that any marginal contribution vector $m^{i}(N, v, L)$ as defined in equation (4.3) is in $C\left(N, v^{L}\right)$. In fact, when $v^{L}$ is superadditive, then $v^{L}$ is permutationally convex for any permutation corresponding to the spanning tree $T^{i}$ and then, according to Granot and Huberman [7], $m^{i}(N, v, L)$ is in $C\left(N, v^{L}\right)$ for all $i \in N$. So, for superadditive games with cycle-free communication structure the average tree solution is in $C(N, v, L)$, because the core is convex. Also for games with cycle-free communication structure, Talman and Yamamoto [18] provide a condition even weaker than superadditivity under which the average tree solution is still in the core. We next introduce the notion of link-convexity, which will be shown to assure that the average tree solution is an element of the core for an arbitrary game with communication structure. 


\section{Definition 5.1 Link-convexity}

A game with communication structure $(N, v, L)$ is link-convex if

$$
v(S)+v(T) \leq v(S \cup T)+\sum_{K \in \widehat{C}^{L}(S \cap T)} v(K),
$$

for any $S, T \subset N$ that satisfy

(1) $S, T, S \backslash T, T \backslash S$, and $(S \backslash T) \cup(T \backslash S)$ are non-empty networks,

(2) $N \backslash S$ or $N \backslash T$ is a network.

Notice that Condition (1) of Definition 5.1 implies that $S \cup T$ is a non-empty network.

Link-convexity reduces to convexity for the class of games with complete communication structure because for those games all subsets of $N$ are networks and convexity is satisfied trivially when $S, T, S \backslash T$, or $T \backslash S$ equals the empty set. We illustrate the concept of link-convexity with an example.

\section{Example 5.2 (Cycle graph)}

We consider the graph $(N, L)$ with $L=\{\{i, i+1\} \mid i=1, \ldots, n\}$, where $n+1=1$, so the players are located on a circle. In this case any non-empty network has form $S=[i, j]$, where $[i, j]$ denotes the set $\{i, i+1, i+2, \ldots, j\}$ if $j \geq i$ and $[i, j]$ denotes the set $\{i, i+1, \ldots, n, 1, \ldots, j\}$ if $j<i$. Observe that for any $S=[i, j]$, the set $N \backslash S$ is a network. By Condition (1) of Definition 5.1 we must have that both $S$ and $T$ are nonempty networks, so for some $i, i^{\prime}, j, j^{\prime}, S=\left[i, i^{\prime}\right]$ and $T=\left[j, j^{\prime}\right]$. Then both $N \backslash S$ and $N \backslash T$ are networks, so Condition (2) of Definition 5.1 is redundant. Without loss of generality assume that $j \geq i$. Then the condition that $S \backslash T$ and $T \backslash S$ are non-empty, requires that $j>i$. Now, if $j \neq i^{\prime}+1$ then we must have that $j^{\prime}=i-1$, otherwise $(S \backslash T) \cup(T \backslash S)$ is not a network. Therefore, for the game with cyclic communication structure the link-convexity property requires that

$$
v(S)+v(T) \leq v(S \cup T)+v(S \cap T)
$$

for all sets $S=\left[i, i^{\prime}\right]$ and $T=\left[j, j^{\prime}\right]$ such that $j>i$ and further $j=i^{\prime}+1$ or $j^{\prime}=i-1$, i.e., the two sets must be such that $S \cup T$ and $S \cap T$ are both networks.

The next theorem shows that the average tree solution is in the core if the game is link-convex.

Theorem 5.3 If the game with communication structure $(N, v, L)$ is link-convex, then $A T(N, v, L) \in C(N, v, L)$. 
Proof. We show that under link-convexity any marginal contribution vector $m^{B}=$ $m^{B}(N, v, L), B \in \mathcal{B}^{L}$, is an element of $C(N, v, L)$, which proves the result because the core is a convex set.

Consider any $B=\left(B_{1}, \ldots, B_{n}\right) \in \mathcal{B}^{L}$. We have by definition of $m^{B}$ that $\sum_{i \in N} m_{i}^{B}=$ $v(N)$. To prove that $\sum_{i \in S} m_{i}^{B} \geq v(S)$ for $S \in C^{L}(N)$, take any $S \in C^{L}(N)$ and let $S_{1}, \ldots, S_{k^{\prime}}$ be the components in the subgraph $\left(S, T^{B}(S)\right)$. Since $S_{k}$ is connected, the directed subgraph $\left(S_{k}, T^{B}\left(S_{k}\right)\right)$ is a tree and there exists a unique $r_{k} \in S_{k}$ such that $S_{k} \subset$ $B_{r_{k}}, k=1, \ldots, k^{\prime}$. Notice that $r_{k}$ is the root of $\left(S_{k}, T^{B}\left(S_{k}\right)\right)$. We define $I=\left\{r_{1}, \ldots, r_{k^{\prime}}\right\}$. By Property (2) of Lemma 3.2, either $B_{i} \subset B_{j} \backslash\{j\}$ or $B_{j} \subset B_{i} \backslash\{i\}$ or $B_{i} \cap B_{j}=\emptyset$ for every pair $i, j \in I$. Since $S$ is a network and because of Property (2) of Lemma 3.2 it holds that there is one node, say, $r_{k^{\prime}}$, such that for every $i \in I \backslash\left\{r_{k^{\prime}}\right\}$ the node $i$ is a subordinate of $r_{k^{\prime}}$ and therefore $B_{i} \subset B_{r_{k^{\prime}}} \backslash\left\{r_{k^{\prime}}\right\}$. Since $S_{i} \subset B_{i}$, for all $i \in I$, we have that $S \subset B_{r_{k^{\prime}}}$.

Next, for $k=1, \ldots, k^{\prime}$, let $F_{r_{k}}=\left\{j \in N \backslash S \mid(i, j) \in T^{B}\right.$ for some $\left.i \in S_{k}\right\}$ be the set of successors of $S_{k}$ in the tree $T^{B}$ outside $S$. We define $J=\cup_{i \in I} F_{i}$ as the set of all such successors. For $j \in J$, let

$$
H_{j}=\left\{i \in I \mid B_{i} \subset B_{j} \text { and } \nexists i^{\prime} \in I \backslash\{i\} \text { such that } B_{i} \subset B_{i^{\prime}} \subset B_{j}\right\} .
$$

We define $\widehat{N}=I \cup J$ and the directed graph $(\widehat{N}, \widehat{T})$ by

$$
\widehat{T}=\left\{(i, j) \mid j \in F_{i}, i \in I\right\} \cup\left\{(j, i) \mid i \in H_{j}, j \in J\right\} .
$$

Clearly, $(\widehat{N}, \widehat{T})$ is a tree with root $r_{k^{\prime}}$.

For $k=1, \ldots, k^{\prime}, B_{r_{k}}=S_{k} \cup\left(\cup_{j \in F_{r_{k}}} B_{j}\right)$, so

$$
\sum_{i \in S} m_{i}^{B}=\sum_{k=1}^{k^{\prime}}\left[v\left(B_{r_{k}}\right)-\sum_{j \in F_{r_{k}}} v\left(B_{j}\right)\right] .
$$

Without loss of generality, let $r_{1}, \ldots, r_{k^{\prime}}$ be such that $k^{1}<k^{2}$ implies $B_{r_{k 1}} \subset B_{r_{k^{2}}}$ or $B_{r_{k^{1}}} \cap B_{r_{k^{2}}}=\emptyset$. Next, for $k=0, \ldots, k^{\prime}$, let $B^{k}=B_{r_{1}} \cup \cdots \cup B_{r_{k}}$, so it follows that $B^{0}=\emptyset$.

Consider some $k \in\left\{1, \ldots, k^{\prime}\right\}$ and write $F_{r_{k}}=\left\{j_{1}, \ldots, j_{\ell^{\prime}}\right\}$. If $F_{r_{k}}=\emptyset$, then we define $l^{\prime}$ to be zero. When $\ell^{\prime} \geq 1$, then, for $\ell=1, \ldots, \ell^{\prime}$, the two sets $S \cup B^{k-1} \cup\left(B_{j_{1}} \cup\right.$ $\cdots \cup B_{j_{\ell-1}}$ ) and $B_{j_{\ell}}$ satisfy Conditions (1) and (2) of Definition 5.1. Notice that the components of their (possibly empty) intersection are the networks $B_{i}$ for $i \in H_{j_{\ell}}$ and that $B_{j_{\ell}} \backslash\left(\cup_{i \in H_{j_{\ell}}} B_{i}\right)$ is linked to $S_{k}$. Now it follows from link-convexity that for $l=1, \ldots, l^{\prime}$,

$$
v\left(S \cup B^{k-1} \cup\left(B_{j_{1}} \cup \cdots \cup B_{j_{\ell-1}}\right)\right)+v\left(B_{j_{\ell}}\right) \leq v\left(S \cup B^{k-1} \cup\left(B_{j_{1}} \cup \cdots \cup B_{j_{\ell}}\right)\right)+\sum_{i \in H_{j_{\ell}}} v\left(B_{i}\right) .
$$

By repeated application of this argument and since $S_{k} \cup B^{k-1} \cup\left(\cup_{j \in F_{r_{k}}} B_{j}\right)=B^{k}$, it follows that for $k=1, \ldots, k^{\prime}$,

$$
v\left(S \cup B^{k-1}\right)+\sum_{j \in F_{r_{k}}} v\left(B_{j}\right) \leq v\left(S \cup B^{k}\right)+\sum_{j \in F_{r_{k}}} \sum_{i \in H_{j}} v\left(B_{i}\right) .
$$


Notice that this formula is also valid if $F_{r_{k}}=\emptyset$, since then $S \cup B^{k-1}=S \cup B^{k}$.

By repeated application of the last inequality, we find that

$$
v(S)+\sum_{k=1}^{k^{\prime}} \sum_{j \in F_{r_{k}}} v\left(B_{j}\right) \leq v\left(S \cup B^{k^{\prime}}\right)+\sum_{k=1}^{k^{\prime}} \sum_{j \in F_{r_{k}}} \sum_{i \in H_{j}} v\left(B_{i}\right) .
$$

Since $S \cup B^{k^{\prime}}=B_{r_{k^{\prime}}}$ and $\widehat{T}$ is a tree, it follows that every $B_{r_{k}}, k=1, \ldots, k^{\prime}-1$, appears exactly once in the right-hand side, and we obtain

$$
v(S)+\sum_{k=1}^{k^{\prime}} \sum_{j \in F_{r_{k}}} v\left(B_{j}\right) \leq \sum_{k=1}^{k^{\prime}} v\left(B_{r_{k}}\right) .
$$

From equations (5.4) and (5.5) it follows that

$$
v(S) \leq \sum_{i \in S} m_{i}^{B}
$$

which completes the proof.

From this theorem the next corollary follows immediately

Corollary 5.4 If a game with communication structure $(N, v, L)$ is link-convex, then the core $C(N, v, L)$ is non-empty.

For games with complete communication structure link-convexity and convexity coincide with each other. The next lemma shows that under weaker conditions than superadditivity, a game with cycle-free communication structure is link-convex.

Lemma 5.5 A game with cycle-free communication structure $(N, v, L)$ is link-convex if and only if for every $S \in C^{L}(N)$ it holds that

$$
v(S)+v(T) \leq v(S \cup T) \text { for all } T \in \widehat{C}^{L}(N \backslash S) .
$$

Proof. Let $S, T \subset N$ satisfy the conditions in Definition 5.1 with $N \backslash T$ being a network. We first show that $S \cap T=\emptyset$. Suppose $S \cap T \neq \emptyset$. Take any $i^{\prime} \in S \cap T$. Since $S \backslash T$ and $T \backslash S$ are non-empty and $(S \backslash T) \cup(T \backslash S)$ is a non-empty network, there exists $i \in S \backslash T$ and $j \in T \backslash S$ such that $\{i, j\} \in L$. Since both $S$ and $T$ are networks, there exists a path in $S$ connecting $i$ and $i^{\prime}$ and there exists a path in $T$ connecting $j$ and $i^{\prime}$. This contradicts the fact that $(N, v, L)$ is a game with cycle-free communication structure.

From $S \cap T=\emptyset$, it follows that $S \subset N \backslash T$. Since both $N \backslash T$ and $S \cup T$ are networks, $S \subset N \backslash T$, and $(N, L)$ is cycle-free, we must have $T \in \widehat{C}^{L}(N \backslash S)$. 
The lemma shows that the condition $v(S)+v(T) \leq v(S \cup T)$ only has to hold for any network $S$ and any network $T$ that is a component of $(N \backslash S, L(N \backslash S))$. Notice that for a game with cycle-free communication structure superadditivity requires that $v(S)+v(T) \leq v(S \cup T)$ for any disjoint $S$ and $T$ such that $S, T$, and $S \cup T$ are networks. So, the next corollary follows immediately.

Corollary 5.6 A game with cycle-free communication structure $(N, v, L)$ is link-convex if $v$ is superadditive.

The following example illustrates that link-convexity is strictly weaker than superadditivity and also that the Myerson value may not be in the core if the game is link-conevex.

\section{Example 5.7 (Path graph)}

We consider the cycle-free graph on $(N, L)$ with $L=\{\{j, j+1\} \mid j=1, \ldots, n-1\}$. In $L$ the players are positioned along a path from 1 to $n$ and any player is connected with his neighbors. In this case any non-empty network $S$ is of the form $S=[i, j]$, $1 \leq i \leq j \leq n$, where $[i, j]=\{i, \ldots, j\}$. From Theorem 5.5 it follows that for a game with path communication structure $(N, v, L)$, link-convexity requires

$$
v(S)+v(T) \leq v(S \cup T)
$$

for all coalitions $S$ and $T$ such that $S=[i, j]$ and $T=[1, i-1]$ or $T=[j+1, k]$. Observe that for games with path communication structure link-convexity is indeed weaker than superadditivity.

For example, consider the path graph with $N=4$ and $L=\{\{1,2\},\{2,3\},\{3,4\}\}$. The values of the connected coalitions are given by $v(\{1\})=v(\{4\})=0, v(\{2\})=2$, $v(\{3\})=4, v([1,2])=v([2,3])=2, v([3,4])=4, v([1,3])=v([2,4])=6$, and $v([1,4])=6$. This game is not superadditive, since for the networks $S=\{2\}$ and $T=\{3\}$ we have that $v([2,3])=2<v(\{2\})+v(\{3\})=6$. Link-convexity only requires that

$$
v([1, j])+v([j+1, k]) \leq v([1, k]) \text { for } j=1,2,3, j+1 \leq k \leq 4
$$

and

$$
v([i, j])+v([j+1,4]) \leq v([i, 4]) \text { for } i=2,3, i \leq j<4 .
$$

Indeed, all these inequalities are satisfied for the game. Observe that this game has a unique core element $(0,2,4,0)^{\top}$.

The average tree solution for this game is equal to the average of the marginal contribution vectors of the spanning trees induced by the four admissible 4-tuples $B^{1}=$ $(N,\{2,3,4\},\{3,4\},\{4\}), B^{2}=(\{1\}, N,\{3,4\},\{4\}), B^{3}=(\{1\},\{1,2\}, N,\{4\})$, and $B^{4}=$ 
$(\{1\},\{1,2\},\{1,2,3\}, N)$. All these 4-tuples yield the same marginal contribution vector, $(0,2,4,0)^{\top}$. Therefore the average tree solution equals $\operatorname{AT}(N, v, L)=(0,2,4,0)^{\top}$, coinciding with the unique core element.

To compute the Myerson value of the game, we first determine the Myerson restricted game $\left(N, v^{L}\right)$. For $S \in C^{L}(N)$ we have $v^{L}(S)=v(S)$ and for $S \notin C^{L}(N)$ we have $v^{L}(\{1,3\})=4, v^{L}(\{1,4\})=0, v^{L}(\{2,4\})=2, v^{L}(\{1,3,4\})=4$, and $v^{L}(\{1,2,4\})=2$.

The Myerson value is equal to the Shapley value of the game $\left(N, v^{L}\right)$ and therefore equal to $\left(\frac{1}{3}, \frac{5}{3}, \frac{11}{3}, \frac{1}{3}\right)^{\top}$. Notice that the Myerson value lies outside the core. This is caused by the fact that the Myerson value is the average of all the 24 marginal vectors of $\left(N, v^{L}\right)$. Each marginal vector of $\left(N, v^{L}\right)$ is induced by a permutation; not every permutation yields a marginal vector which is a core element. For example, the permutation $\pi^{1}=(1,4,2,3)$ leads to the marginal vector $m^{\pi^{1}}=(0,-2,4,4)^{\top}$ and the permutation $\pi^{2}=(4,1,3,2)$ leads to the marginal vector $m^{\pi^{2}}=(0,4,0,2)^{\top}$. Both marginal vectors lie outside the core and are not induced by an admissible 4-tuple of coalitions.

\section{Concluding remarks}

In this paper the average tree solution is proposed for the class of all games with communication structure. This solution generalizes both the solution introduced by Herings et al. [9] for the class of games with cycle-free communication structure and the Shapley value for the class of games with complete communication structure. We introduce the condition of link-convexity under which the average tree solution is an element of the core. For the class of games with cycle-free communication structure, link-convexity is weaker than superadditivity. In general, link-convexity is weaker than convexity, and only coincides with it for games with complete communication structure.

Following this study, Baron et al. [1] define and axiomatize the average tree solution for any class of spanning trees. They also investigate several properties of the average tree solution. In particular, they prove that the set of spanning trees induced by the class of admissible $n$-tuples of coalitions is the largest class of spanning trees satisfying that the corresponding average tree solution has the Harsanyi property.

\section{References}

[1] Baron, R., S. Béal, E. Rémilla, and P. Solal (2008), Average Tree Solutions for Graph Games, Preprint.

[2] Borm, P., G. Owen, and S. Tijs (1992), On the Position Value for Communication Situations, SIAM Journal of Discrete Mathematics, 5, 305-320. 
[3] Brink, R. van den, G. van der Laan, and V. Pruzhansky (2004), Harsanyi Power Solutions for Graph-Restricted Games, TI Discussion Paper 2004-095/1, Tinbergen Institute, Amsterdam/Rotterdam.

[4] Demange, G. (1994), Intermediate Preferences and Stable Coalition Structures, Journal of Mathematical Economics, 23, 45-58.

[5] Demange, G. (2004), On Group Stability in Hierarchies and Networks, Journal of Political Economy, 112, 754-778.

[6] Gillies, D.B. (1953), Some Theorems on n-Person Games, Princeton University Press, Princeton.

[7] Granot, G., and G. Huberman (1982), The Relationship Between Convex Games and Minimal Cost Spanning Tree Games: A Case for Permutationally Convex Games, SIAM Journal of Algebraic and Discrete Methods, 3, 288-292.

[8] Harsanyi, J.C. (1959), A Bargaining Model for Cooperative $n$-Person Games, in A.W. Tucker and R.D. Luce (eds.), Contributions to the Theory of Games IV, Princeton University Press, Princeton, pp. 325-355.

[9] Herings, P.J.J., G. van der Laan, and A.J.J. Talman (2008), The Average Tree Solution for Cycle-free Graph Games, Games and Economic Behavior, 62, 77-92.

[10] Kaneko, M., and M.H. Wooders (1982), Cores of Partitioning Games, Mathematical Social Sciences, 3, 313-327.

[11] Le Breton, M., G. Owen, and S. Weber (1992), Strongly Balanced Cooperative Games, International Journal of Game Theory, 20, 419-427.

[12] Meessen, R. (1988), Communication Games, Master Thesis (in Dutch), University of Nijmegen, Nijmegen.

[13] Myerson, R.B. (1977), Graphs and Cooperation in Games, Mathematics of Operations Research, 2, 225-229.

[14] Myerson, R.B. (1980), Conference Structures and Fair Allocation Rules, International Journal of Game Theory, 9, 169-182.

[15] Owen, G. (1986), Values of Graph-Restricted Games, SIAM Journal on Algebraic and Discrete Methods, 7, 210-220. 
[16] Shapley, L. (1953), A Value for n-Person Games, in H.W. Kuhn and A.W. Tucker (eds.), Contributions to the Theory of Games II, Princeton University Press, Princeton, pp. 307-317.

[17] Slikker, M. (2005), A Characterization of the Position Value, International Journal of Game Theory, 33, 505-514.

[18] Talman, A.J.J. and Y. Yamamoto (2007), Games with Limited Communication Structure, CentER Discussion paper 2007-19, CentER, Tilburg University, Tilburg, forthcoming in Journal of the Operation Research Society of Japan.

[19] Velzen, S. van (2005), Cooperation in Network and Scheduling, PhD Thesis, CentER, Tilburg University, Tilburg. 